\title{
Neuroticism and Emotional Risk During the COVID-19 Pandemic
}

\author{
Kroencke, L. ${ }^{1 *}$, Geukes, K. ${ }^{1}$, Utesch, T. ${ }^{1}$, Kuper, N. ${ }^{2}$, \& Back, M. D. ${ }^{1}$ \\ ${ }^{1}$ University of Münster, Germany, ${ }^{2}$ Bielefeld University, Germany
}

\section{Journal of Research in Personality, in press}

This is an unedited manuscript accepted for publication. The manuscript will undergo copyediting, typesetting, and review of resulting proof before it is published in its final form.

\footnotetext{
Author Note

L.K., K.G., T.U., and M.D.B. conceptualized and designed the study. L.K. ran the data collection. L.K., T.U., and N.K. analyzed the data. L.K., K.G., and M.D.B. wrote the manuscript. All authors edited and approved the submitted manuscript.

We embrace the values of openness and transparency in science (Schönbrodt et al., 2015; osf.io/4dvkw/). We therefore follow the 21-word solution (Simmons et al., 2012), or refer to project documentations in the OSF. We furthermore publish all raw data necessary to reproduce reported results and provide scripts for all data analyses reported in this manuscript (see http://go.wwu.de/t7hy9). We are grateful to Matthias P. Walther, Martje Kohlhoff, Tina N. Ricken, and Steffen Filz for their help collecting the data and to Tabea Straeten for her help with data documentation.

${ }^{*}$ Correspondence concerning this article should be addressed to L. Kroencke (lara.kroencke@wwu.de), University of Münster, Institute for Psychology, Psychological Assessment and Personality Psychology, Fliednerstr. 21, 48149 Münster, Germany.
} 


\begin{abstract}
Large-scale health crises, such as the COVID-19 pandemic, may evoke negative affective responses, which are linked to psychological maladjustment and psychopathology. Here, we shed light on the role of the personality trait neuroticism in predicting who experiences negative affective responses. In a large-scale experience-sampling study $(N=1,609 ; 38,120$ momentary reports), we showed that individuals high in neuroticism experienced more negative affect and higher affective variability in their daily lives. Individuals high in neuroticism also (a) paid more attention to COVID-19-related information and worried more about the consequences of the pandemic (crisis preoccupation), and (b) experienced more negative affect during this preoccupation (affective reactivity). These findings offer new insights into the consequences and dynamics of neuroticism in extreme environmental contexts.
\end{abstract}

Keywords: negative affect, neuroticism, personality, COVID-19, worry, experience sampling 


\section{Neuroticism and Emotional Risk During the COVID-19 Pandemic}

Large-scale health crises, such as the COVID-19 pandemic, pose serious health threats to members of affected societies. Humans generally respond to such threats with negative affect, which may lead to lower psychological well-being and psychopathology (Brooks et al., 2020; Wichers, 2014). To identify and support individuals with negative affective responses, policymakers and journalists typically emphasize the role of sociodemographic factors (e.g., advanced age, living alone). Previous research has, however, shown that individual differences in personality predict psychological well-being beyond these aspects (Boyce et al., 2013). The present research aims to evaluate the relationship between personality and negative affect in an extreme environmental context that should strongly trigger individual differences in affective functioning. We focus on neuroticism, a personality trait that has far-reaching public health significance (Lahey, 2009). First, we examine whether neuroticism is associated with negative affect level and variability. Second, we investigate whether neuroticism is related to (a) higher attention to COVID-19-related information and higher engagement in COVID-19-related worries (crisis preoccupation), and (b) stronger negative affect during this preoccupation (affective reactivity).

\section{Neuroticism and Negative Affect}

Neuroticism refers to individual differences in negative emotionality (Barlow et al., 2014; Lahey, 2009) and predicts a range of negative outcomes, including low subjective well-being (Lucas, 2018; Soto, 2019) and poor physical and mental health (Kotov et al., 2010; Malouff et al., 2005). In previous experience-sampling studies that repeatedly asked individuals about their everyday life experiences, individuals with high levels of neuroticism reported more negative affect on average (i.e., higher negative affect levels). In some studies, individuals with high levels of neuroticism also varied more in their in negative affect from moment to moment (Geukes et al., 2017) - although the robustness of these findings is currently a subject of debate (Hisler et al., 2020; Kalokerinos et al., 2020; Wendt et al., 2020). Please note that variability refers to fluctuations around a general tendency and does not consider temporal dependency (EbnerPriemer et al., 2009). Thus, these studies examined the association of neuroticism with the degree to which individuals showed similar levels of negative affect across situations (low variability) versus strong differences in negative affect across situations (high variability), independent of their temporal ordering. 
The COVID-19 pandemic represents an extreme situation that poses unique mental health challenges (Galea et al., 2020; Holmes et al., 2020), and it is unclear how the relationships between neuroticism and negative affect level and variability unfold during a pandemic. Some have argued that individual differences in personality should be studied during environmental challenges because novel and uncertain situations are more likely to reveal the effects of dispositional tendencies on people’s everyday experiences and behaviors (Caspi \& Moffitt, 1993). This argument is backed up by dynamic theories of personality (Denissen \& Penke, 2008; Tett \& Guterman, 2000), which posit that individual differences in personality should be more consequential in situations that activate the traits. Following this logic, the COVID-19 pandemic, which is marked by stressors such as threat and uncertainty, represents a prototypical situation to study the effects of neuroticism on everyday negative affect. Alternatively, the COVID-19 situation may induce extreme situational pressures that obliterate the relationship between neuroticism and negative affect (Cooper \& Withey, 2009). Examining the effects of neuroticism on negative affect level and variability during the COVID-19 pandemic, thus, does not only allow to identify a potentially important but overlooked predictor of negative affect during the crisis, it also allows to test basic principles of personality expression in extreme environmental contexts.

First studies have examined well-being and mental health symptoms during the COVID19 pandemic (Rajkumar, 2020; Thombs et al., 2020) and identified risk groups based on sociodemographic variables (Lai et al., 2020; Wang et al., 2020). Importantly, these studies captured well-being in decontextualized ways and cannot speak to the effects on negative affect as it is experienced in people's daily lives, fluctuating from moment to moment and embedded into actual situations. The few existing experience-sampling studies that investigated fluctuations in negative affect during the COVID-19 pandemic (e.g., Fried et al., 2020; Huckins et al., 2020; Stieger et al., 2020) were all comparatively small ( $N=80,286$, and 217 , respectively), mostly focused on specific populations (e.g., college students), and did not examine personality predictors of negative affective states. Based on these gaps in the literature, a first goal of this research is to provide robust empirical evidence to resolve whether and how strongly neuroticism predicts negative affect level and variability in this unprecedented situation.

\section{Crisis Preoccupation and Affective Reactivity}

To better understand the relationship between neuroticism and negative affect during the COVID-19 pandemic, we further zoom in on two processes that have been linked to neuroticism before COVID-19: crisis preoccupation and affective reactivity. First, previous research has 
shown that individuals high in neuroticism are prone to rumination and worry, which are both crucially involved in mental health problems (Merino et al., 2016; Muris et al., 2005). Prior to the pandemic, these worries may have centered around various issues other than COVID-19 (e.g., occupational, interpersonal, or financial problems). Here, we aim to examine whether the worries also attach themselves to pandemic-related content. Specifically, we investigate whether individuals high in neuroticism pay more attention to COVID-19-related information and worry more about the consequences of the pandemic (e.g., for their own health; crisis preoccupation). Because repetitive negative thoughts are linked to psychological distress (Watkins, 2008), crisis preoccupation may contribute to heightened levels of negative affect.

A second process that has been linked to neuroticism before the COVID-19 pandemic is affective reactivity to stress (Bolger \& Schilling, 1991). The neuroticism - stress-reactivity relationship is backed up by daily diary studies, in which individuals high in neuroticism reacted more negatively to stressful events (Hisler et al., 2020; Howland et al., 2017; Suls \& Martin, 2005). During the COVID-19 pandemic, attention to COVID-19 related information and healthrelated worries may act as cognitive stressors that trigger negative affective reactions, particularly in individuals high in neuroticism (i.e., affective reactivity). Stronger affective reactivity may contribute to both heightened levels of negative affect and heightened affective variability. Yet, most previous studies on the neuroticism - stress-reactivity relationship focused on objective daily stressors and only few studies have examined individual differences in affective reactivity in response to cognitive preoccupation (Segerstrom et al., 2020). In addition, previous investigations of real-life preoccupation and affective reactivity processes took place outside of an immediate crisis context. A second goal of this research is, thus, to provide a high-powered investigation of crisis preoccupation and affective reactivity processes in a directly relevant context.

\section{The Present Study}

This study uses cutting-edge experience-sampling methodology (Myin-Germeys et al., 2009; Wright \& Zimmermann, 2019) to examine the relationship between neuroticism and (a) negative affect level and variability, and (b) crisis preoccupation and affective reactivity during the early phases of the COVID-19 pandemic in Germany (March 18 - April 3, 2020). The COVID-19 pandemic reached Germany on January 27 when the first person in Bavaria was infected with the virus. The virus quickly spread to other states and on March 8, the first death due to COVID-19 was reported in Germany. On March 11, the World Health Organization 
declared COVID-19 a global health pandemic (World Health Organization, 2020). In Germany, first nationwide restrictions were announced on March 16, including travel bans, the closure of kindergartens, schools, universities, and many non-essential stores and facilities (e.g., bars, clubs, discos, theatres, museums, concert halls, cinemas, zoos, leisure parks, sports facilities, playgrounds). These restrictions led to severe disruptions of people’s everyday lives. Many people were forced to work from home, homeschool their children, and to physically distance themselves from others. On March 18, the day when the data collection started, Chancellor Angela Merkel called COVID-19 Germany’s greatest challenge since World War II. The restrictions were further intensified on March 22, when the German government announced a decree of restrained contact to prohibit gatherings of more than two people from different households. In addition, the study period (March 18 - April 3, 2020) was marked by a rapid increase of infections and deaths from 10,999 to 79,696 and 20 to 1,017, respectively (Robert Koch Institute, 2020a, 2020b).

During this period, a large sample of participants $(N=1,609)$ initially completed an online survey on demographic, personality, and individual COVID-19-related information (e.g., personally experienced health threats). They then provided fine-grained, longitudinal measures of their daily activities over the course of 2 weeks. Analyses focused in the relationships between neuroticism and (a) negative affect level and variability, and (b) crisis preoccupation and affective reactivity.

Our study seeks to extend previous work by (a) examining the role of neuroticism during an unprecedented health crisis, (b) capturing both the level and variability in negative affect, and (c) focusing on crisis preoccupation and affective reactivity processes. It thus allows for robust insights that are needed to (a) derive scientifically informed strategies for dealing with major health crises, and (b) test theoretical principles on the consequences and dynamics of neuroticism in high stakes contexts.

\section{Method}

In this Method section, we describe the sample and relevant procedures and measures of the data collection project. A codebook with a full description of all procedures and measures applied in this project as well as all data and analytical code necessary to reproduce reported results can be retrieved from http://go.wwu.de/t7hy9. All procedures used in this study were approved by the review board of MASKED FOR REVIEW. None of the tested hypotheses was preregistered. 


\section{Participants}

Data for the present paper were provided by a German convenience sample consisting of 1,609 participants (78\% women, $M_{\text {age }}=33.7$ years, $S D_{\text {age }}=12.7$, Range $\left.=16-83\right)$. Most participants (84\%) held at least an A-level (German Abitur), and most of them were either working (51\%) or attending a university program (37\%). Participants were recruited via media announcements and received personalized feedback in May 2020 on their emotional states as an incentive for participation.

\section{Procedures}

The study was programmed in formR version v0.18.3 (Arslan et al., 2020) and released on March 18, 2020. The study began when social distancing measures were recommended but not yet officially advised (March 18) and covered critical events such as the decree of restrained contact on March 22 and the decree of prolonged restrained contact on April 1, as well as marked increases in deaths due to COVID-19 (from 20 deaths on March 19 to 1,017 deaths on April 3).

Data collection consisted of three phases: an initial and a final online survey and an experience-sampling phase in between. For the initial online survey (Day 1), participants provided informed consent and information on demographic variables (e.g., age, sex, cohabitation status, educational status, occupational status), on personality traits (e.g., neuroticism), and on their experiences during the COVID-19 pandemic (e.g., personally experienced health threats). The subsequent experience-sampling phase (Days 2 to 15) began the next day in the morning. Participants completed up to six surveys per day for a maximum of 14 days. Each survey assessed momentary states (e.g., negative affect, attention to COVID-19related information, and engagement in COVID-19-related worries) and took about 2 minutes to complete. Participants received an email reminder for each survey that provided them with a personalized link to the respective survey. The emails were sent at random time points throughout the day based on individual time preferences for daily start and end times, with the stipulation that two successive surveys had to be a minimum of 40 minutes apart. Surveys were accessible for 45 minutes after the email reminder was sent. If participants did not respond to a reminder within 20 minutes, one additional reminder was sent. The first item of every ESM survey asked participants whether they had engaged in a social interaction (> 5 minutes) since the last survey. If participants answered "yes”, all subsequent questions referred to their most recent social interaction. If they answered "no", all subsequent questions referred to their most recent individual activity. An individual activity was defined as the last situation that lasted at least 5 
minutes and during which the participant did not interact with others (see http://go.wwu.de/t7hy9 for the original wording of all measures). The link to the final online survey was sent the day after the experience-sampling phase was completed (Day 16). In this online survey, participants provided (additional) information on demographic variables (i.e., relationship status, information about social networks) as well as on personality traits and their experiences during the COVID19 pandemic.

We aimed to collect as many participants as possible within the critical time period to obtain precise estimates of effect sizes. For the present analyses, we included participants $(N=$ 1,609) with the initial and the experience-sampling surveys who provided state data on or before April 3, 2020. This cut-off date was chosen as most participants had started within the first three days of the study. The number of completed surveys per day decreased strongly after April 3, as fewer participants were still enrolled. Moreover, we included data on the final COVID-19 survey for participants ( $n=686$ ) who completed the whole study on or before April 4, 2020, that is, who started within the first three days of the study. Surveys were excluded in cases of partial completion. On average, participants of this study took part for 7.4 days $(M d n=7)$ and completed 3.2 surveys $(M d n=3)$ per day, with a total average of 23.7 surveys $(M d n=15)$ per participant. Note that our final sample includes a high number of participants who completed only one $(n=158)$, two $(n=115)$, or three $(n=109)$ surveys. Because our focus is on dynamic changes within individuals and thus requires repeated measurements, we reran all analyses with a subsample of participants who provided at least 10 assessments $(n=942$; total $=35,664$ momentary reports).

\section{Measures}

Whenever items were aggregated to form a composite score, we either computed a mean per participant (trait measures) or a mean per time point per participant (state measures).

\section{Sociodemographic Information (Trait Survey)}

For the sociodemographic variables, we assessed age, sex, educational status, occupational status, and participants' household size. The last variable was recoded into a dummy variable that indicated whether or not participants were living with others or alone (cohabitation status). Moreover, participants indicated whether (Yes) or not (No) their personal social network included grandparents, parents, siblings, their own children, and a partner. 
Participants completed the Neuroticism subscale from the German version of the Big Five Inventory-2 S (Rammstedt et al., 2018). This scale comprises six items, with two items assessing the neuroticism facets anxiety, depression, and emotional volatility, respectively. These items were answered on 5-point rating scales with the anchors: 1 (disagree strongly), 2 (disagree a little), 3 (neutral; no opinion), 4 (agree a little), and 5 (agree strongly).

\section{Personally Experienced Health Threats (Trait Survey)}

To assess the extent to which participants were personally affected by the COVID-19 pandemic, they completed a total of six items. Four were used in the present study because they were deemed theoretically most relevant and exhibited considerable variance between individuals. These items were answered on a dichotomous response format (Yes vs. No) and targeted different degrees of personal health threat. The items read: “Are you in voluntary quarantine?” “Are you in mandatory quarantine?” “Do you have or did you have the typical

disease symptoms (e.g., fever, cough)?” and “Do you think one or more people in your household are part of the risk group for the Coronavirus?” We formed a composite score reflecting the degree of experienced health threats by summing (a) whether participants were in either voluntary or mandatory quarantine, (b) whether participants had the typical disease symptoms (e.g., fever, cough), and (c) whether participants thought that one or more people in their household belonged to the risk group for COVID-19.

\section{Negative Affect (State Survey)}

Participants rated their emotional states on a total of 16 items. Three items were included as indicators of negative affect for this study on the basis of their theoretical relevance. Depending on whether or not participants had engaged in a social interaction ( $>5$ minutes) since the last survey, the item stem read either "How did you feel directly after the interaction?” or "How did you feel directly after the activity?” The items themselves were "anxious," "relaxed” (reverse coded), and “insecure.” These items were answered on 6-point rating scales ranging from 1 (do not agree at all) to 6 (agree completely) and aggregated on the basis of conceptual reasons.

\section{Attention to COVID-19-related Information (State Survey)}

To assess attention to COVID-19-related information, participants completed different versions of one item, depending on whether or not they had engaged in a social interaction (> 5 minutes) since the last survey. If participants reported a social interaction, the item stem read "During the interaction, I showed the following behavior:" and the item read "I addressed the 
topic of Coronavirus." When there had been no social interaction, the item stem read "During the activity, I experienced the following:” and the item read "I thought about the Coronavirus.” Both items were answered on 6-point rating scales ranging from 1 (do not agree at all) to 6 (agree completely).

\section{COVID-19-related Worries (State Survey)}

To assess COVID-19-related worries, participants completed a total of 14 items, all of which had the item stem “Because of the spread of the Coronavirus, I worry about...” and were answered on a rating scale from 1 (very little) to 6 (very much). Three items targeted worries about oneself. The item wordings were “...my health,” “...my social life,” and “...my studies/work life.” Three items referred to worries about “my parents’ health,” “...my close friends’ health,” and “...the health of members of my wider social environment (classmates, other acquaintances).” These items were aggregated to form an index of worries about others' health. Four additional items were aggregated to form an index of worries about societal issues, that is, “...the health system in Germany,” “...the social cohesion in Germany,” “...the economy/work life in Germany,” and “...the cultural life in Germany.” Finally, worries about one’s own health, one’s social life, one’s work/studies, others' health, and societal issues were averaged to form an aggregate of different types of worry.

\section{Analytical Strategy}

For the statistical analyses, all continuous variables were $z$-standardized across the entire dataset. Sex, cohabitation status, the presence versus absence of social interactions, and weekend versus weekday were dummy-coded. Educational status and occupational status were treated as categorical variables. All analyses were run in $R$ (R Core Team, 2018) or Mplus 8 (Muthén \& Muthén, 2017).

In a first step, we investigated the between-person correlations of all main variables included in our analyses, using the R-package psych (Revelle, 2018). Next, we ran multilevel models with the R-package nlme (Pinheiro et al., 2020), in which neuroticism predicted participants' random intercepts in negative affect. We used three-level models with measurement occasions nested in days and days nested in participants and included a residual autocorrelation for consecutive measurement occasions within the same day. Moreover, we controlled for several between-person (age, sex, cohabitation status, educational status, occupational status, personally experienced health threats) and time-varying variables (presence vs. absence of social interactions, weekend vs. weekday, time). 
To examine the relationships between neuroticism and variability in negative affect, we ran multilevel location scale models. These models represent an extension of standard multilevel models, in which the residual terms are allowed to vary between individuals (Geukes et al., 2017). Both random intercepts (representing mean levels) and random residual variances (representing within-person variability) were modelled as latent variables, which were allowed to covary. In the first model, neuroticism was used as a between-person predictor of the random intercepts and residual variances. The results reflect the correlations between neuroticism and negative affect level und variability, respectively. In the second model, the random intercepts and residual variances were regressed onto neuroticism simultaneously to examine their unique associations with neuroticism. The results can be interpreted as the semi-partial or part correlations between neuroticism and negative affect level und variability, respectively (Hisler et al., 2020). All models were estimated in Mplus using the default, uninformed priors. The parameter estimates were based on two chains with 5,000 iterations each (2,500 iterations were discarded as burn-in). Variability was modelled as the log-transformed residual variance to preclude negative values. The Mplus code was adapted from McNeish (2020) and can be retrieved from our OSF page (http://go.wwu.de/t7hy9).

In a second step, we examined the associations between negative affect, attention to COVID-19, and worries about one's own health, with a particular focus on whether neuroticism moderated these associations (affective reactivity). To this end, we specified similar multilevel models as described above (i.e., three levels, autocorrelated residuals). Whenever worries or attention to COVID-19 served as an independent variable, they were disaggregated into their within-person (WP) and between-person (BP) parts. To this end, we included two predictors in our models: the person mean (BP predictor) and the time-specific deviation from the person mean (WP predictor). The within-person effects were allowed to vary between participants. Affective reactivity was assessed as the cross-level interaction between neuroticism and attention to COVID-19 and between neuroticism and worries about one's own health in the prediction of negative affect, respectively. Moreover, the interaction between neuroticism and attention to COVID-19 in the prediction of worries about one's own health was examined. In all three models, we examined whether neuroticism was related to both the within- and between-person effect. These analyses were repeated by simultaneously including the between-person and timevarying control variables described above. Due to the high number of statistical tests, we used an alpha level of .01 (two-sided) for all tests and report 99\%-confidence intervals. 
We conducted four sets of robustness analyses. First, we examined whether our results regarding worries about one's own health generalized to a broad worry aggregate including five worry variables. Second, we investigated whether our findings were robust to log-transforming negative affect, because the distribution of negative affect is often skewed. Third, we repeated the affective reactivity analyses with the other Big Five traits (extraversion, agreeableness, conscientiousness, openness) and with the three neuroticism facets anxiety, depression, and emotional volatility. Finally, the correlational analyses, variability and affective reactivity analyses were repeated based on a subsample including only those participants who provided at least 10 valid momentary reports.

To gauge our statistical power to detect our effects of interest, a simulation-based power analysis was carried out. To this end, we focused on two models: (1) the model predicting negative affect from worries about one's own health, neuroticism, and their interaction, and (2) the model predicting negative affect from attention to COVID-19, neuroticism, and their interaction (see Table 3). Our simulation yielded power estimates for effect sizes of varying magnitudes and generally indicated that power was good even for the detection of relatively small effects (i.e., $B=0.05$ ). For full details, see Tables S1 and S2 in the Supplementary Materials.

\section{Results}

Table 1 shows descriptive information for the main variables, including intraclass correlations and reliabilities.

\section{Neuroticism and Negative Affect}

First, we analyzed whether neuroticism predicted negative affect level and variability during the COVID-19 pandemic in Germany. In line with our hypotheses, neuroticism was associated with higher mean levels of negative affect in daily life, $r(1607)=0.51[0.46,0.56], p<$ .001 (see Fig. 1 and Supplementary Table S3). Neuroticism predicted negative affect levels beyond a wide range of control variables. These included person-level variables, such as demographic information (age, sex, cohabitation status, educational status, occupational status) and personally experienced health threats (being in quarantine, having symptoms, living with a person from a risk group), as well as time-varying predictors, namely, the presence versus absence of social interactions, whether it was a weekend versus weekday, and the linear effect of time (Supplementary Table S4). Notably, neuroticism predicted individual differences in negative 
affect better than all the control variables combined (neuroticism: $R^{2}=0.26$ vs. control variables: $R^{2}=0.03$; Supplementary Table S5).

\section{Table 1}

Descriptive Statistics

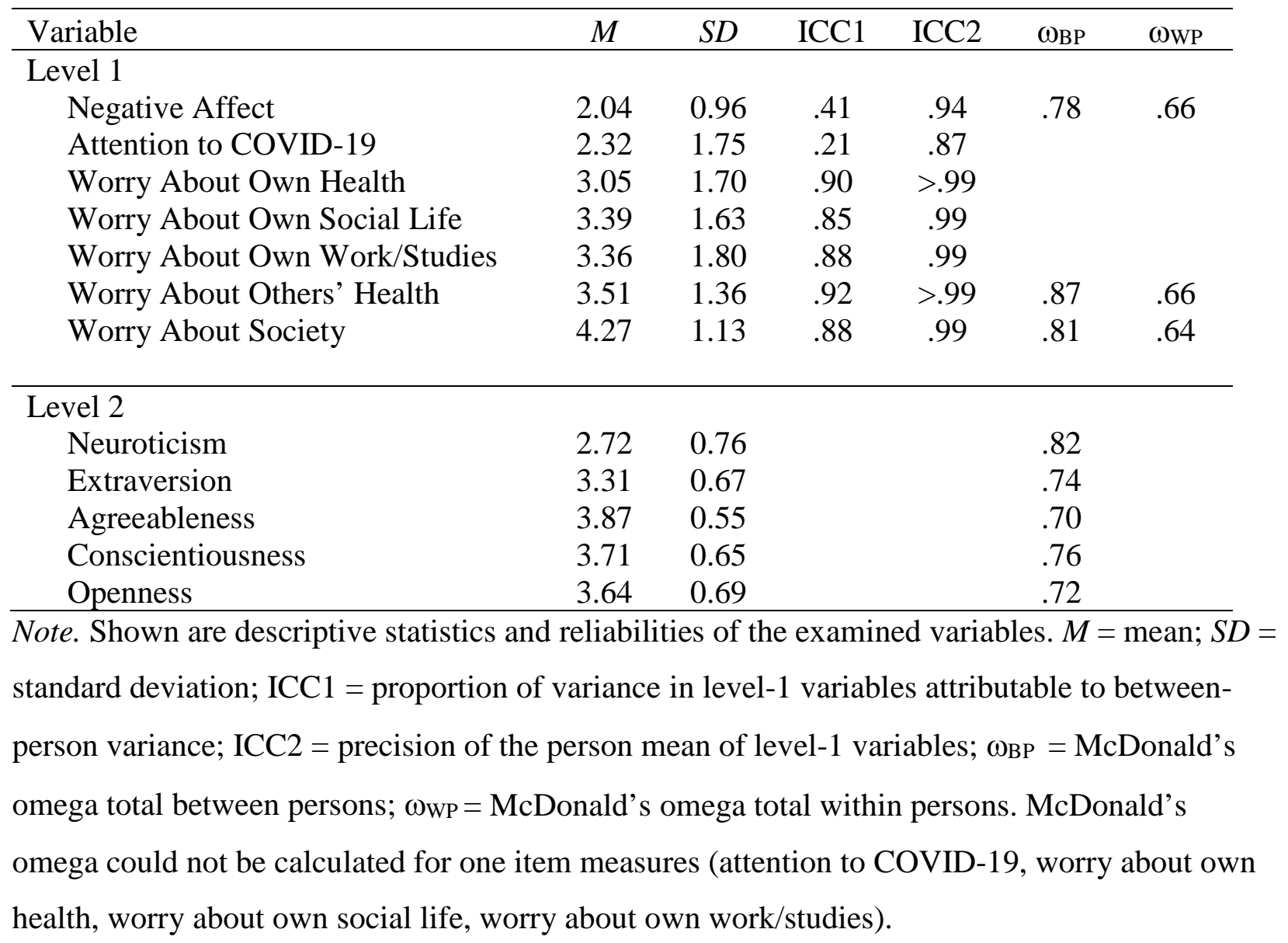




\section{Figure 1}

\section{Illustration of Main Effects of Neuroticism}

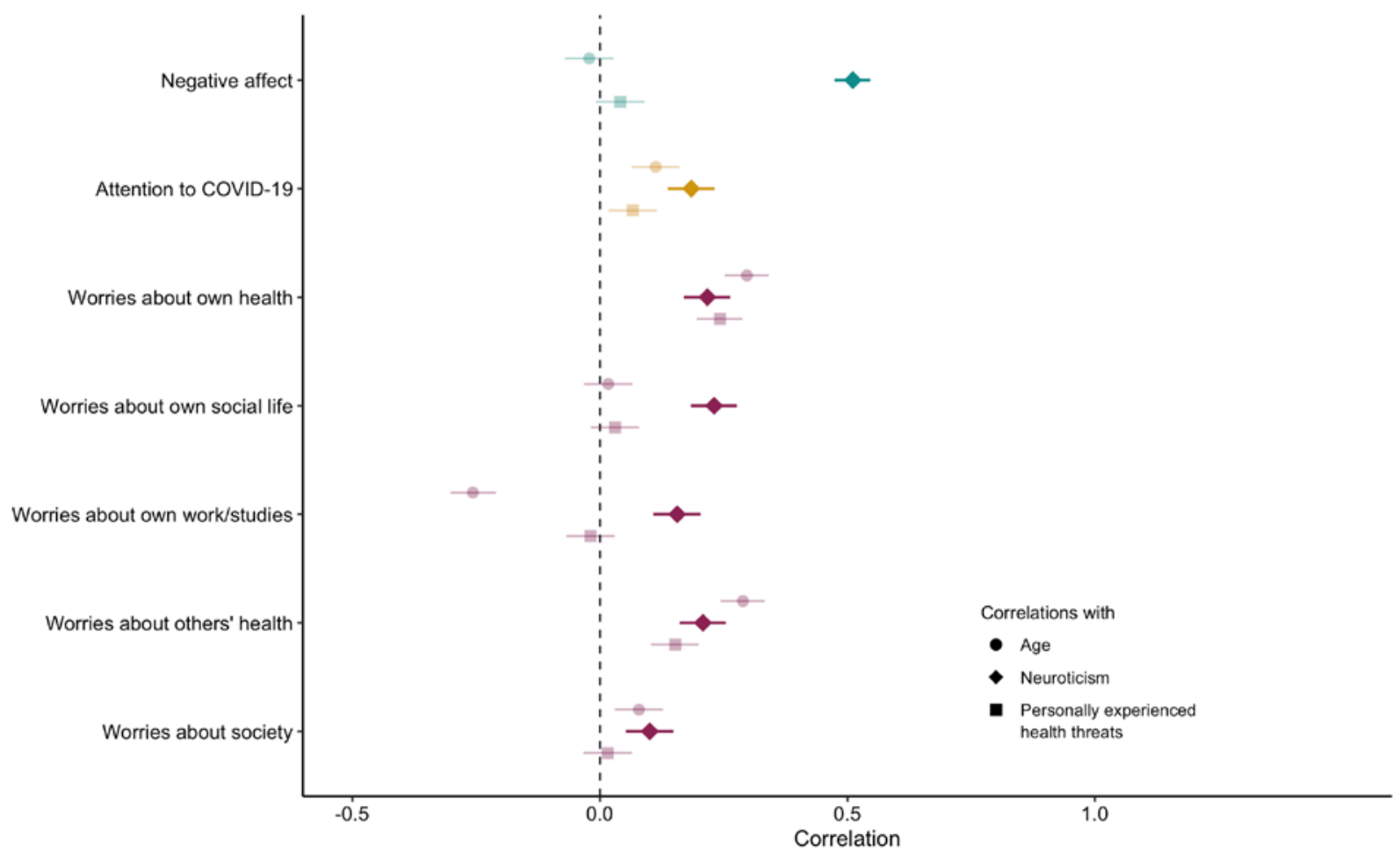

Note. Relationships of neuroticism, age, and personally experienced health threats with negative affect, attention to COVID-19, and worries. Each correlation is shown as a point estimate and 95\%-confidence interval.

In multilevel location scale models, neuroticism was not only related to individual differences in mean levels of negative affect, but also to individual differences in within-person variability. Individuals with high levels of neuroticism experienced more negative affect overall $(b=0.380$ [0.344, 0.415], $p<.001)$ and also varied more in their negative affective experiences ( $b=0.284$ [0.245, 0.322], $p<.001$; Table 2). The relationship between neuroticism and affective variability was robust to alternative modeling strategies such as controlling for mean levels (Hisler et al., 2020), suggesting that neuroticism was uniquely related to both affective characteristics (see Table 2). These findings highlight neuroticism's importance as a predictor of negative affect during major health crises. 
Table 2

Neuroticism and Negative Affect Mean Levels and Variability

\begin{tabular}{lccc}
\hline & Estimate & Post. SD & $p$ \\
\hline Model 1: Neuroticism Predicting Affective Characteristics & & \\
Neuroticism $\rightarrow$ Mean Level & 0.380 & 0.018 & 0.000 \\
Neuroticism $\rightarrow$ Variability & 0.284 & 0.020 & 0.000 \\
Mean Level $\leftrightarrow$ Variability & 0.581 & 0.021 & 0.000 \\
Model 2: Regressing Neuroticism on Multiple Affective Characteristics Simultaneously \\
Mean Level $\rightarrow$ Neuroticism & 0.431 & 0.034 & 0.000 \\
Variability $\rightarrow$ Neuroticism & 0.091 & 0.037 & 0.008 \\
Mean Level $\leftrightarrow$ Variability & 0.655 & 0.018 & 0.000 \\
\hline
\end{tabular}

Note. Shown are standardized estimates from multilevel location scale models. All models were estimated using Bayesian estimation procedures with uninformed priors. Variability was modelled as the log-transformed residual variance. Post. $S D=$ posterior standard deviation. $P$ values are one-sided.

The longitudinal course of negative affect is illustrated in Figure 2. For the entire 2-week period, levels of negative affect were low to moderate for the average person, even lower for individuals low in neuroticism, but considerably elevated for individuals high in neuroticism.

\section{Crisis Preoccupation and Affective Reactivity}

Second, we examined neuroticism's associations with mean levels of attention and worries (crisis preoccupation). As shown in Figure 1 and Supplementary Table S3, neuroticism was related to paying more attention to COVID-19-related information, $r(1607)=0.18$ [0.12, $0.25], p<.001$, and worrying more about one's own health, $r(1607)=0.22[0.16,0.28], p<.001$. We focused on worrying about one's own health because such worries are most clearly conceptually linked to intrapersonal neuroticism processes and the salience of health issues during pandemics. However, the effects were similar for other COVID-19-related worries (see Fig. 1 and Supplementary Table S3). 


\section{Figure 2}

\section{Illustration of Longitudinal Effects of Neuroticism}

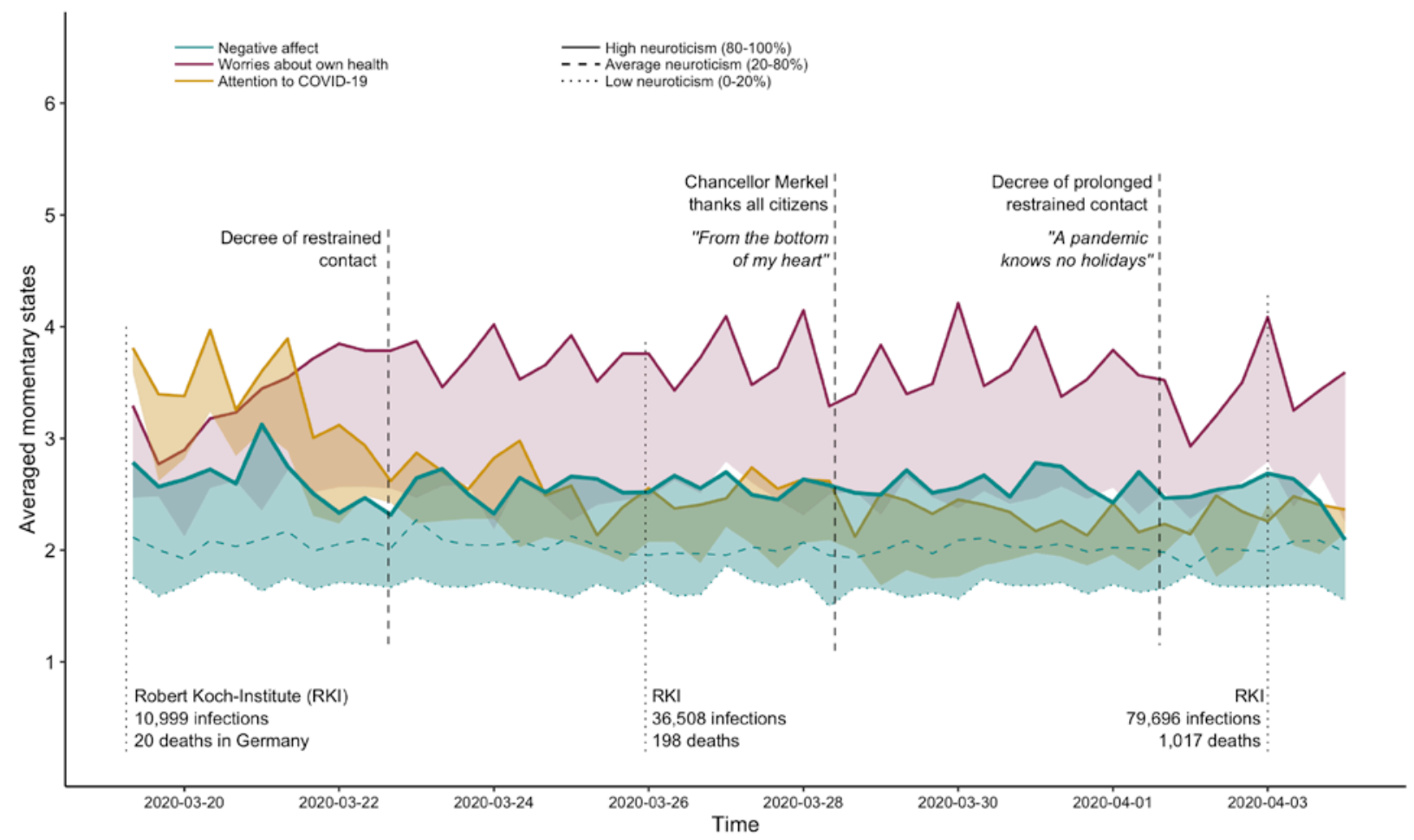

Note. Longitudinal course of negative affect, attention to COVID-19, and worries over the 2week period for individuals with high, medium, and low levels of neuroticism. The lower bound of the shaded areas is the typical trajectory for an individual low in neuroticism (percentile 20) and the upper bound is the typical trajectory for an individual high in neuroticism (percentile 80). All items were answered on 6-point rating scales ranging from 1 (do not agree at all) to 6 (agree completely).

As depicted in Figure 2, attention was highest in the beginning of the 2-week period and decreased thereafter. Worries about one’s own health increased between March 20 and March 22 (decree of restrained contact) and remained at a high level for the rest of the study. Individuals high in neuroticism consistently paid more attention to COVID-19-related information and worried more about its consequences for their own health.

Finally, we investigated whether neuroticism moderated how strongly attention and worries were related to negative affect (affective reactivity). In multilevel models (see Table 3), both attention to COVID-19-related information and worries about one's own health were related to more negative affect in daily life on the within-person $(b=0.187[0.166,0.207], p<.001$ and 
$b=0.221$ [0.163, 0.279], $p<.001$, respectively) and between-person level $(b=0.339$ [0.281, 0.397], $p<.001$ and $b=0.156$ [0.114, 0.198], $p<.001$, respectively). Attention and worries were mutually related on the within-person $(b=0.019[0.012,0.026], p<.001)$ and the betweenperson level $(b=0.185$ [0.101, 0.270], $p<.001)$. In line with the idea of affective reactivity, neuroticism moderated the within-person relationships between attention to COVID-19-related information and negative affect $(b=0.037$ [0.016, 0.057], $p<.001)$ and between worries about one's own health and negative affect ( $b=0.072$ [0.014, 0.129], $p=.001$ ) but not between attention and worries $(b=0.000$ [-0.007, 0.007], $p=.986)$. In addition, neuroticism moderated the between-person relationship of attention to COVID-19 and negative affect $(b=0.116$ [0.061, 0.172], $p<.001)$.

The effects of neuroticism were substantial in size: An examination of the beta coefficients suggested that the within-person effects of attention and worry on negative affect were approximately 50\% (attention) and 95\% (worry) higher for those scoring 1 standard deviation above the mean in neuroticism compared with those scoring 1 standard deviation below the mean. The results remained significant when controlling for demographic variables, personally experienced health threats, the presence versus absence of social interactions, weekend versus weekday, and time (Supplementary Table S6). Moreover, the results were similar when examining an aggregate of different types of worry (Supplementary Table S7) and when logtransforming negative affect (Supplementary Table S8).

As part of our sensitivity analyses, we examined the effects of the other Big Five traits (see Supplementary Tables S9-S14) and the effects of the three neuroticism facets anxiety, depression, and emotional volatility separately. The facet findings were similar to the findings for the full trait measure (see Supplementary Tables S15-S18). Lastly, we reran the main analyses for the most compliant participants (at least 10 assessments; $n=942$; total $=35,664$ momentary reports). As shown in Supplementary Tables S19-S21, the results did not change substantially. Our main findings are visually represented in Figure 3. 


\section{Table 3}

Neuroticism and Affective Reactivity

\begin{tabular}{|c|c|c|c|c|}
\hline Predictor & Estimate & Std. Error & $t$ & $p$ \\
\hline \multicolumn{5}{|c|}{ Model 1: Worry About Own Health Predicting Negative Affect } \\
\hline Neuroticism & 0.282 & 0.015 & 18.265 & 0.000 \\
\hline Worry About Own Health WP & 0.221 & 0.023 & 9.795 & 0.000 \\
\hline Worry About Own Health BP & 0.156 & 0.016 & 9.534 & 0.000 \\
\hline Neuroticism * Worry About Own Health WP & 0.072 & 0.022 & 3.220 & 0.001 \\
\hline Neuroticism * Worry About Own Health BP & 0.026 & 0.015 & 1.722 & 0.085 \\
\hline \multicolumn{5}{|c|}{ Model 2: Attention to COVID-19 Predicting Negative Affect } \\
\hline Neuroticism & 0.269 & 0.015 & 18.045 & 0.000 \\
\hline Attention to COVID-19 WP & 0.187 & 0.008 & 23.445 & 0.000 \\
\hline Attention to COVID-19 BP & 0.339 & 0.023 & 15.029 & 0.000 \\
\hline Neuroticism * Attention to COVID-19 WP & 0.037 & 0.008 & 4.646 & 0.000 \\
\hline Neuroticism * Attention to COVID-19 BP & 0.116 & 0.021 & 5.400 & 0.000 \\
\hline \multicolumn{5}{|c|}{ Model 3: Attention to COVID-19 Predicting Worry About Own Health } \\
\hline Neuroticism & 0.175 & 0.024 & 7.268 & 0.000 \\
\hline Attention to COVID-19 WP & 0.019 & 0.003 & 6.933 & 0.000 \\
\hline Attention to COVID-19 BP & 0.185 & 0.033 & 5.659 & 0.000 \\
\hline Neuroticism * Attention to COVID-19 WP & -0.000 & 0.003 & -0.018 & 0.986 \\
\hline Neuroticism * Attention to COVID-19 BP & 0.007 & 0.031 & 0.241 & 0.810 \\
\hline
\end{tabular}

Note. The results are based on multilevel models (3 levels: time points nested in days nested in persons) with autoregressive errors and random slopes. All variables were z-standardized. Models including control variables can be found in the Supplementary Materials online. WP = withinperson; BP = between-person. 


\section{Figure 3}

\section{Illustration of Moderating Effects of Neuroticism}

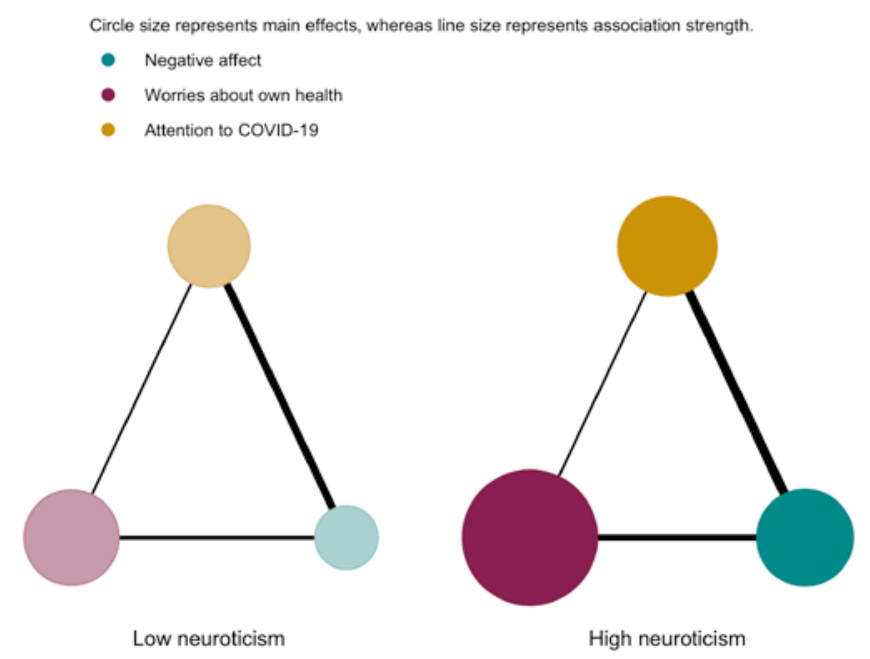

Note. Networks showing the relationships between attention to COVID-19, worries, and negative affect. High = percentile: $80-100$; low = percentile: $0-20$.

\section{Discussion}

This study shows that global pandemics threaten not only people’s physical well-being but also their psychological well-being, particularly for individuals high in neuroticism. In line with findings from representative samples (Entringer \& Kröger, 2020), we observed no dramatic increases in negative affect on average. However, there were substantial differences between individuals, and these differences were closely related to neuroticism. Whereas negative affect was low to moderate for most people, people high in neuroticism experienced elevated levels of negative affect throughout the entire study period. The relationship between neuroticism and negative affect was higher than that of socio-demographic variables and personally experienced health threats combined, underlining neuroticism's public health significance (Lahey, 2009). Contrary to previous studies (Hisler et al., 2020), neuroticism was also uniquely related to higher affective variability. This suggests that during an immediate crisis situation, individuals high in neuroticism experience more negative affect on average and vary more in their negative affect.

In a second step, we identified crisis preoccupation and affective reactivity as two central neuroticism processes in crises situations: Individuals high in neuroticism attend to and worry more about COVID-19-related information (crisis preoccupation) and both attention and worry are linked to more negative affect (affective reactivity). These processes may be mutually reinforcing, such that attention to COVID-19 is linked to more worries and negative affect, which 
in turn are related to more attention. Our findings on neuroticism processes both complement and extend past investigations in several ways. First, our results are in line with pre-pandemic findings on the relationship of neuroticism with rumination and worry (Merino et al., 2016; Muris et al., 2005). We extend previous studies by showing that, during COVID-19, negative repetitive thoughts in individuals high in neuroticism are likely to center around COVID-19-related issues. Second, our results dovetail with existing work on neuroticism and stress reactivity, which has shown that people high in neuroticism react more strongly to daily stressful events (Hisler et al., 2020; Howland et al., 2017; Suls \& Martin, 2005), including internal, cognitive stressors such as worries (Segerstrom et al., 2020). The present study showed that individuals high in neuroticism also react more negatively to internal, cognitive stressors triggered by major health crises.

Our study was unique because we tested theoretical principles on the consequences and dynamics of neuroticism during an immediate crisis situation. Thus, besides replicating previous findings in a well-powered and intensive study, we showed that findings from previous research also apply to extreme environmental contexts. The fact that neuroticism was uniquely linked to higher variability suggests that neuroticism processes may even be enhanced during times of crises. However, based on the present data, we cannot identify whether the relationships between attention and worries with negative affect were accentuated compared to pre-COVID-19 times because we did not have a pre-pandemic baseline. Future studies should compare the relationships between neuroticism, attention, worries, and negative affect before vs. during COVID-19 to delineate which aspects of neuroticism processes have changed as a response to the pandemic.

It is important to note that our investigation provided a rather conservative test of these hypotheses as this study took place in Germany, a country that was only mildly affected by the COVID-19 pandemic during the study period. Therefore, our study may have underestimated the absolute levels of negative affectivity, the effects of neuroticism, and the strength of the underlying processes. It will be important to replicate our findings in other countries that are more seriously affected by the pandemic. Moreover, as our investigation took place during the first weeks of the outbreak, the focus was on short-term stressors and their immediate consequences. Future studies should examine whether the effects reported here weaken or accumulate over time and whether crisis preoccupation and affective reactivity are related to long-term mental health consequences. Lastly, our sample covered a wide age range (16-83) and was thus relatively diverse compared to student samples. Nevertheless, we would like to highlight 
that participants selected themselves into the sample, which may have led to a selection bias (Schaurer \& Weiß, 2020).

Our findings have important implications for applied settings. Identifying groups who experience negative affectivity on the basis of sociodemographic information alone may overlook people in need of help, such as individuals high in neuroticism. Neuroticism is typically assessed via short self-report scales (Rammstedt et al., 2018) but new technologies even allow for the assessment of neuroticism on the basis of digital footprints (e.g., likes on social media platforms). These technologies could be used to screen large numbers of individuals with minimal effort and to deliver personality-tailored prevention campaigns (e.g., targeting individuals high in neuroticism with information on crisis preoccupation and affective reactivity processes; Matz et al., 2017). Delivering personality-tailored prevention strategies, while considering ethical standards regarding data privacy (Matz et al., 2020), may help to prevent increases in mental health problems due to the COVID-19 and future pandemics (Galea et al., 2020). To implement significant and scientifically informed prevention campaigns, policymakers should actively seek out psychological policy consultation, and psychological scientists should explicitly and responsibly communicate their findings to the public (Bleidorn et al., 2019). 


\section{References}

Arslan, R. C., Walther, M. P., \& Tata, C. S. (2020). formr: A study framework allowing for automated feedback generation and complex longitudinal experience-sampling studies using R. Behavior Research Methods, 52, 376-387.

Barlow, D. H., Sauer-Zavala, S., Carl, J. R., Bullis, J. R., \& Ellard, K. K. (2014). The nature, diagnosis, and treatment of neuroticism: Back to the future. Clinical Psychological Science, 2, 344-365.

Bleidorn, W., Hill, P. L., Back, M. D., Denissen, J. J. A., Hennecke, M., Hopwood, C. J., Jokela, M., Kandler, C., Lucas, R. E., Luhmann, M., Orth, U., Wagner, J., Wrzus, C., Zimmermann, J., \& Roberts, B. (2019). The policy relevance of personality traits. American Psychologist, 74, 1056-1067.

Bolger, N., \& Schilling, E. A. (1991). Personality and the problems of everyday life: The role of neuroticism in exposure and reactivity to daily stressors. Journal of Personality, 59, 355386.

Boyce, C. J., Wood, A. M., \& Powdthavee, N. (2013). Is personality fixed? Personality changes as much as "variable” economic factors and more strongly predicts changes to life satisfaction. Social Indicators Research, 111, 287-305.

Brooks, S. K., Webster, R. K., Smith, L. E., Woodland, L., Wessely, S., Greenberg, N., \& Rubin, G. J. (2020). The psychological impact of quarantine and how to reduce it: Rapid review of the evidence. The Lancet, 395, 912-920.

Caspi, A., \& Moffitt, T. E. (1993). When do individual differences matter? A paradoxical theory of personality coherence. Psychological Inquiry, 4, 247-271.

Cooper, W. H., \& Withey, M. J. (2009). The strong situation hypothesis. Personality and Social Psychology Review, 13, 62-72.

Denissen, J. J. A., \& Penke, L. (2008). Motivational individual reaction norms underlying the Five-Factor model of personality: First steps towards a theory-based conceptual framework. Journal of Research in Personality, 42, 1285-1302.

Ebner-Priemer, U. W., Eid, M., Kleindienst, N., Stabenow, S., \& Trull, T. J. (2009). Analytic strategies for understanding affective (in)stability and other dynamic processes in psychopathology. Journal of Abnormal Psychology, 118, 195-202.

Entringer, T., \& Kröger, H. (2020). Einsam, aber resilient - Die Menschen haben den Lockdown besser verkraftet als vermutet. DIW Berlin - Deutsches Institut für Wirtschaftsforschung. 
Fried, E. I., Papanikolaou, F., \& Epskamp, S. (2020, April 24). Mental health and social contact during the COVID-19 pandemic: An ecological momentary assessment study. https://doi.org/10.31234/osf.io/36xkp

Galea, S., Merchant, R. M., \& Lurie, N. (2020). The mental health consequences of COVID-19 and physical distancing: The need for prevention and early intervention. JAMA Internal Medicine, 180, 817-818.

Geukes, K., Nestler, S., Hutteman, R., Küfner, A. C., \& Back, M. D. (2017). Trait personality and state variability: Predicting individual differences in within- and cross-context fluctuations in affect, self-evaluations, and behavior in everyday life. Journal of Research in Personality, 69, 124-138.

Hisler, G. C., DeHart, T., Krizan, Z., \& Wright, A. G. (2020). Neuroticism as the intensity, reactivity, and variability in day-to-day affect. Journal of Research in Personality, 87, 103964.

Holmes, E. A., O'Connor, R. C., Perry, V. H., Tracey, I., Wessely, S., Arseneault, L., Ballard, C., Christensen, H., Silver, R. C., Everall, I., \& Ford, T. (2020). Multidisciplinary research priorities for the COVID-19 pandemic: A call for action for mental health science. The Lancet Psychiatry, 7, 547-560.

Howland, M., Armeli, S., Feinn, R., \& Tennen, H. (2017). Daily emotional stress reactivity in emerging adulthood: Temporal stability and its predictors. Anxiety, Stress, \& Coping, 30, 121-132.

Huckins, J., Hedlund, E. L., Rogers, C., Nepal, S. K., Wu, J., Obuchi, M., Murphy, E. I., Meyer, M. L., Wagner, D. D., Holtzheimer, P. E., \& Campbell, A. T. (2020, May 4). Mental health and behavior during the early phases of the COVID-19 pandemic: A longitudinal mobile smartphone and ecological momentary assessment study in college students. https://doi.org/10.2196/20185

Kalokerinos, E. K., Murphy, S. C., Koval, P., Bailen, N. H., Crombez, G., Hollenstein, T., Gleeson, J., Thompson, R. J., Van Ryckeghem, D. M. L., Kuppens, P., \& Bastian, B. (2020). Neuroticism may not reflect emotional variability. Proceedings of the National Academy of Sciences, 117, 9270-9276.

Kotov, R., Gamez, W., Schmidt, F., \& Watson, D. (2010). Linking “big” personality traits to anxiety, depressive, and substance use disorders: A meta-analysis. Psychological Bulletin, 136, 768-821. 
Lahey, B. B. (2009). Public health significance of neuroticism. American Psychologist, 64, 241256.

Lai, J., Ma, S., Wang, Y., Cai, Z., Hu, J., Wei, N., Wu, J., Du, H., Chen, T., Li, R., \& Tan, H. (2020). Factors associated with mental health outcomes among health care workers exposed to coronavirus disease 2019. JAMA Network Open, 3, e203976.

Lucas, R. E. (2018). Exploring the associations between personality and subjective well-being. In E. Diener, S. Oishi, \& L. Tay (Eds.), Handbook of Well-Being (pp. 257-271). DEF Publishers.

Malouff, J. M., Thorsteinsson, E. B., \& Schutte, N. S. (2005). The relationship between the fivefactor model of personality and symptoms of clinical disorders: A meta-analysis. Journal of Psychopathology and Behavioral Assessment, 27, 101-114.

Matz, S. C., Appel, R. E., \& Kosinski, M. (2020). Privacy in the age of psychological targeting. Current Opinion in Psychology, 31, 116-121.

Matz, S. C., Kosinski, M., Nave, G., \& Stillwell, D. J. (2017). Psychological targeting as an effective approach to digital mass persuasion. Proceedings of the National Academy of Sciences, 114, 12714-12719.

McNeish, D. (2020). Specifying location-scale models for heterogeneous variances as multilevel SEMs. Organizational Research Methods. Advance online publication. https://doi.org/10.1177/1094428120913083

Merino, H., Senra, C., \& Ferreiro, F. (2016). Are worry and rumination specific pathways linking neuroticism and symptoms of anxiety and depression in patients with generalized anxiety disorder, major depressive disorder and mixed anxiety-depressive disorder? PloS One, 11, e0156169.

Muris, P., Roelofs, J., Rassin, E., Franken, I., \& Mayer, B. (2005). Mediating effects of rumination and worry on the links between neuroticism, anxiety and depression. Personality and Individual Differences, 39, 1105-1111.

Muthén, L. K., \& Muthén, B. O. (1998-2017). Mplus User’s Guide. Eighth Edition. Los Angeles: Muthén \& Muthén.

Myin-Germeys, I., Oorschot, M., Collip, D., Lataster, J., Delespaul, P., \& Van Os, J. (2009). Experience sampling research in psychopathology: Opening the black box of daily life. Psychological Medicine, 39, 1533-1547. 
Pinheiro, J., Bates, D., DebRoy, S., Sarkar, D., \& R Core Team (2020). Nlme: linear and nonlinear mixed effects models. R package version 3.1-145

R Core Team. (2018). R: A language and environment for statistical computing. R Foundation for Statistical Computing, Vienna, Austria. https://www.r-project.org/

Rajkumar, R. P. (2020). COVID-19 and mental health: A review of the existing literature. Asian Journal of Psychiatry, 52, 102066.

Rammstedt, B., Danner, D., Soto, C. J., \& John, O. P. (2018). Validation of the short and extrashort forms of the Big Five Inventory-2 (BFI-2) and their German adaptations. European Journal of Psychological Assessment, 36, 149-161.

Revelle, W. (2018). Psych: procedures for personality and psychological research. R package version 1.9.12.31

Robert Koch Institute (2020a). Täglicher Lagebericht des RKI zur Coronavirus-Krankheit-2019 (COVID-19): 19.03.2020.

https://www.rki.de/DE/Content/InfAZ/N/Neuartiges_Coronavirus/Situationsberichte/2020 -03-19-de.pdf?_blob=publicationFile

Robert Koch Institute (2020b). Täglicher Lagebericht des RKI zur Coronavirus-Krankheit-2019 (COVID-19): 03.04.2020.

https://www.rki.de/DE/Content/InfAZ/N/Neuartiges_Coronavirus/Situationsberichte/2020 -04-03-de.pdf?_blob=publicationFile

Schaurer, I., \& Weiß, B. (2020). Investigating selection bias of online surveys on coronavirusrelated behavioral outcomes. Survey Research Methods, 14, 103-108.

Schönbrodt, F. D., Maier, M., Heene, M., \& Zehetleitner, M. (2015). Voluntary commitment to research transparency. http://www.researchtransparency.org

Segerstrom, S. C., Gloger, E. M., Hardy, J. K., \& Crofford, L. R. (2020). Exposure and reactivity to repetitive thought in the neuroticism-distress relationship. Cognitive Therapy and Research, 44, 659-667.

Simmons, J. P., Nelson, L. D., \& Simonsohn, U. (2012). A 21 Word Solution. http://dx.doi.org/10.2139/ssrn.2160588

Soto, C. J. (2019). How replicable are links between personality traits and consequential life outcomes? The Life Outcomes of Personality Replication Project. Psychological Science, 30, 711-727. 
Stieger, S., Lewetz, D., \& Swami, V. (2020, May 26). Psychological well-being under conditions of lockdown: An experience sampling study in Austria during the COVID-19 pandemic. https://doi.org/10.31234/osf.io/qjhfp

Suls, J., \& Martin, R. (2005). The daily life of the garden-variety neurotic: Reactivity, stressor exposure, mood spillover, and maladaptive coping. Journal of Personality, 73, 14851510.

Tett, R. P., \& Guterman, H. A. (2000). Situation trait relevance, trait expression, and crosssituational consistency: Testing a principle of trait activation. Journal of Research in Personality, 34, 397-423.

Thombs, B. D., Bonardi, O., Rice, D. B., Boruff, J. T., Azar, M., He, C., Markham, S., Sun, Y., Wu, Y., Krishnan, A., \& Thombs-Vite, I. (2020). Curating evidence on mental health during COVID-19: A living systematic review. Journal of Psychosomatic Research, 133, 110113.

Wang, C., Pan, R., Wan, X., Tan, Y., Xu, L., Ho, C. S., \& Ho, R. C. (2020). Immediate psychological responses and associated factors during the initial stage of the 2019 Coronavirus Disease (COVID-19) epidemic among the general population in China. International Journal of Environmental Research and Public Health, 17, 1729.

Watkins, E. R. (2008). Constructive and unconstructive repetitive thought. Psychological Bulletin, 134, 163-206.

Wendt, L. P., Wright, A. G., Pilkonis, P. A., Woods, W. C., Denissen, J. J. A., Kühnel, A., \& Zimmermann, J. (2020). Indicators of affect dynamics: Structure, reliability, and personality correlates. European Journal of Personality. Advance online publication. https://doi.org/10.1002/per.2277

Wichers, M. (2014). The dynamic nature of depression: A new micro-level perspective of mental disorder that meets current challenges. Psychological Medicine, 44, 1349-1360.

World Health Organization (2020, March 11). WHO Director-General's opening remarks at the media briefing on COVID-19 - 11 March 2020.

https://www.who.int/dg/speeches/detail/who-director-general-s-opening-remarks-at-themedia-briefing-on-covid-19---11-march-2020

Wright, A. G., \& Zimmermann, J. (2019). Applied ambulatory assessment: Integrating idiographic and nomothetic principles of measurement. Psychological Assessment, 31, 1467-1480. 